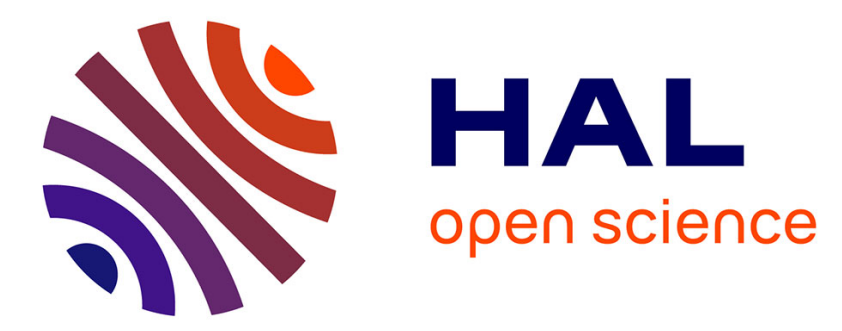

\title{
Détermination analytique de la résistance électrique de barreaux rectangulaires - 2e partie: Dispositifs cylindriques infinis à trois électrodes latérales
}

\author{
A. Morand, J. Robert
}

\section{- To cite this version:}

A. Morand, J. Robert. Détermination analytique de la résistance électrique de barreaux rectangulaires - 2e partie: Dispositifs cylindriques infinis à trois électrodes latérales. Revue de Physique Appliquée, 1981, 16 (5), pp.249-257. 10.1051/rphysap:01981001605024900 · jpa-00244917

\section{HAL Id: jpa-00244917 https://hal.science/jpa-00244917}

Submitted on 1 Jan 1981

HAL is a multi-disciplinary open access archive for the deposit and dissemination of scientific research documents, whether they are published or not. The documents may come from teaching and research institutions in France or abroad, or from public or private research centers.
L'archive ouverte pluridisciplinaire HAL, est destinée au dépôt et à la diffusion de documents scientifiques de niveau recherche, publiés ou non, émanant des établissements d'enseignement et de recherche français ou étrangers, des laboratoires publics ou privés. 


\title{
Détermination analytique de la résistance électrique de barreaux rectangulaires
}

\section{$2^{\mathrm{e}}$ partie : Dispositifs cylindriques infinis à trois électrodes latérales}

\author{
A. Morand et J. Robert \\ Laboratoire de Génie Electrique des Universités Paris VI et Paris XI, E.S.E., Plateau du Moulon, 91190 Gif sur Yvette, France
}

(Reçu le 12 janvier 1981, révisé le 5 février 1981, accepté le 6 février 1981)

\begin{abstract}
Résumé. - Le prësent article est consacré à la détermination des résistances interélectrodes de barreaux rectangulaires cylindriques infinis à trois électrodes latérales. Toute disposition d'électrodes est ramenée à une forme unique à l'aide de transformations conformes. La détermination des résistances interélectrodes, sous la forme d'un schéma équivalent en triangle, fait intervenir la notion de géométries complémentaires et repose sur l'emploi de deux abaques. Le premier permet d'évaluer deux résistances sur les trois. La dernière s'exprime en fonction des précédentes et d'une longueur caractéristique mise en évidence par la métallisation d'un intervalle entre électrodes. Cette longueur est déterminée, de manière approximative, à l'aide du second abaque. L'approximation est évaluée en annexe. La méthode est appliquée à la détermination des dimensions d'un barreau à trois électrodes correspondant à un schéma équivalent donné, ainsi qu'à l'évaluation de la résistance d'un barreau à deux électrodes quelconques présentant un saillant métallisé ou une entaille isolante.
\end{abstract}

\begin{abstract}
The interelectrode resistances are determined in three-electrode infinite rectangular systems. For any location of the electrodes the system is turned into a basic shape by means of conformal mappings. The three interelectrode resistances, pertaining to a delta equivalent circuit, are determined by means of two charts. The first one gives the value of two resistances over the three. The last resistance is related to the others and to a characteristic length featured by the metallization of the interval between two electrodes. This length is given, approximately, by the second chart. The approximation is evaluated in the appendix. The process is applied to the determination of the characteristic lengths in a three-electrode system with a known equivalent circuit, and to the evaluation of the electrical resistance in a two-electrode system exhibiting a metallized indentation or an insulating notch.
\end{abstract}

Le présent travail constitue la seconde partie d'un article paru dans cette revue [1]. Des rappels préliminaires permettent de lire œ qui suit de manière indépendante.

1. Rappels. - Les systèmes étudiés sont des cylindres infinis de section droite rectangulaire. Le plan d'une section droite est identifié au plan complexe $(x, y)$ de façon à introduire le potentiel complexe

$$
W(z)=U(x, y)+j V(x, y) .
$$

Les fonctions $U$ et $V$ sont respectivement la fonction flux et le potentiel. On appelle plan de référence le plan complexe $(\xi, \eta)$ tel que

$$
W=\xi+j \eta \text {. }
$$

Les plans complexes $(x, y)$ et $(\xi, \eta)$ sont associés par une transformation conforme, notée $(T)$.
Pour un quadrilatère curviligne quelconque du plan complexe $(x, y)$ délimité par deux lignes de courant et deux équipotentielles, la résistance électrique $R$, réduite par rapport à la résistance par carré, est

$$
R=\frac{\Delta V}{\Delta U}
$$

où $\Delta V$ et $\Delta U$ désignent respectivement les variations de $V$ et de $U$. On appelle géométrie complémentaire d'une géométrie donnée celle qui est obtenue en permutant les fonctions $U$ et $V$. Sa résistance réduite $R^{\prime}$ est liée à la résistance $R$ du dispositif initial par l'expression

$$
R R^{\prime}=1
$$

2. Dispositifs à trois électrodes. - On considère un barreau cylindrique infini, constitué par un matériau résistant homogène, portant latéralement trois élec- 
trodes infinies quelconques. La section droite rectangulaire du barreau est représentée sur la figure $1 a$. Cette géométrie est soumise aux transformations conformes successives de la figure 1. La première est une transformation de Schwarz-Christoffel [2] qui associe l'intérieur du rectangle au demi-plan Y positif (Fig. 1b). Cette transformation correspond à la fonction sinus elliptique ayant pour argument la variable complexe $z$ et pour paramètre le nombre $m$ qui est une fonction du rapport des côtés du rectangle. Ensuite une transformation homographique (Fig. 1c) permet d'obtenir deux espaces interélectrodes symétriques par rapport à l'axe des ordonnées. Enfin une nouvelle transformation de Schwarz-Christoffel conduit au rectangle de la figure $1 d$. Le paramètre $m^{\prime}$ qui caractérise ce nouveau rectangle se déduit de la transformation homographique précédente. Dans la suite de l'étude, un barreau à trois électrodes quelconques sera représenté directement sous la forme de la figure $1 d$.

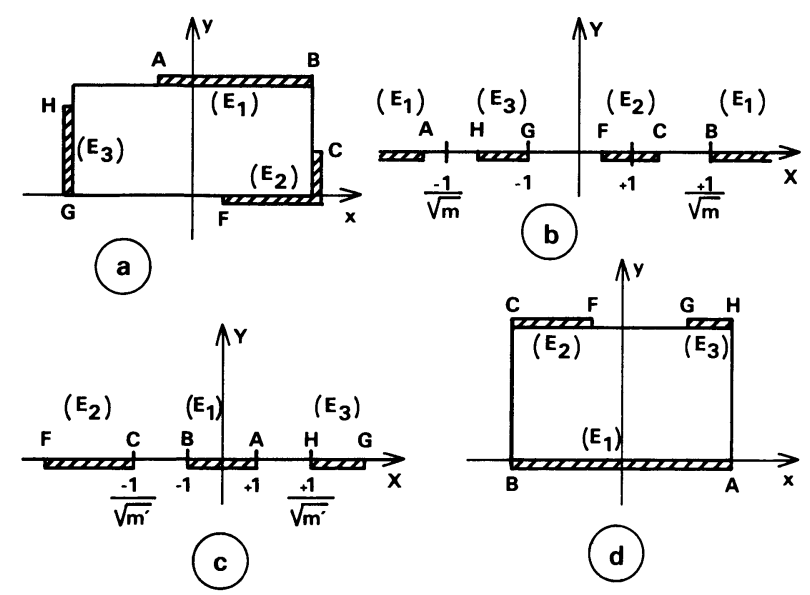

Fig. 1. - Transformations de dispositifs rectangulaires. a) Section droite d'un barreau cylindrique infini portant latéralement trois électrodes métalliques quelconques. b) Dispositif issu du cas $a$ par application d'une transformation de Schwarz-Christoffel. $c$ ) Dispositif à symétrie partielle déduit du cas $b$ par une transformation homographique. d) Rectangle associé au cas précédent par une nouvelle transtormation de Schwarz-Christoffel.

[Transformations of rectangular systems. a) Cross section of an infinite cylindrical bar. Three metallic electrodes, of any size or location, are fitted on the side of the homogeneous resistive material. b) System derived from (a) by a Schwarz-Christoffel transformation. The parameter $m$ value is related to the ratio of the rectangle sides. $c)$ Partly symmetrical system derived from $(b)$ by a bilinear transformation. d) Rectangle related to (c) by another Schwarz-Christoffel transformation. 7

3. Résistance interélectrodes. - Les résistances interélectrodes d'un dispositif à trois électrodes (Fig. 2a) peuvent être définies à l'aide d'un schéma équivalent en triangle (Fig. $2 b$ ) ou en étoile (Fig. $2 c$ ). La méthode de détermination de ces résistances fait intervenir le dispositif complémentaire, obtenu par permutation des électrodes et des intervalles entre électrodes (Fig. 2d). Entre les représentations en triangle et en étoile d'un système donné existent les relations :

$$
R_{i 0}=\frac{R_{i j} R_{i k}}{R_{\mathrm{P}}} \quad R_{i j}=\frac{R_{i 0} R_{j 0}}{R_{\mathrm{E}}}
$$

avec $R_{\mathrm{P}}=\sum R_{i j} ; \quad\left(R_{\mathrm{E}}\right)^{-1}=\sum\left(R_{i 0}\right)^{-1}$

$$
(i, j, k=1,2,3 \text { et } i \neq j \neq k) \text {. }
$$
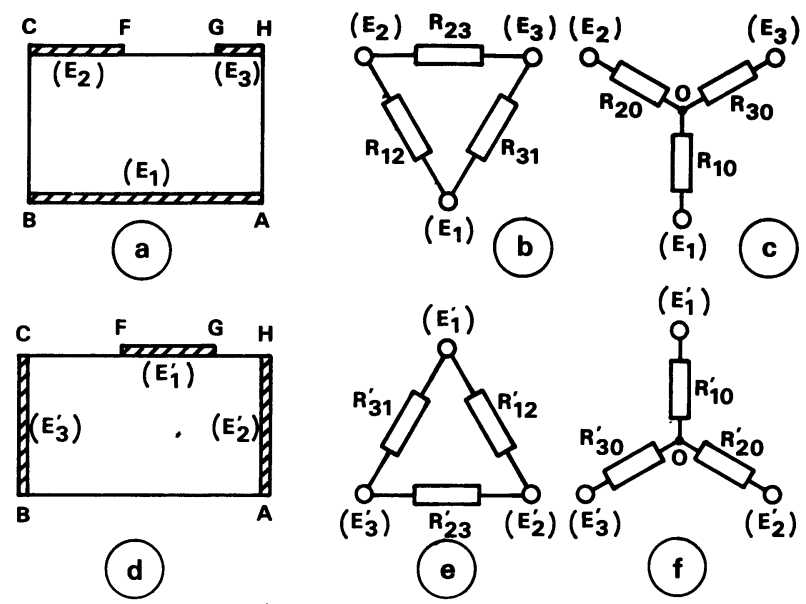

Fig. 2. - Schémas équivalents. a) Tripôle constitué par un barreau rectangulaire à trois électrodes. $b$ ) et $c$ ) Dispositions en triangle et en étoile des résistances interélectrodes du tripôle précédent. $d$ ) Dispositif complémentaire du cas $(a)$. $e$ ) et $f$ ) Schémas équivalents en triangle et en étoile du tripôle précédent.

[Equivalent circuits. a) Three-pole system constituted by a rectangle with three electrodes. $b$ ) and $c$ ) Delta and star connections for the interelectrode resistances of the above system. $d$ ) Complementary system of $(a)$. It results from the interchange between the electrodes and their spacings. $e$ ) and $f$ ) Delta and star equivalent circuits for $(c)$.]

Il existe en outre des relations entre les résistances de deux dispositifs complémentaires. Pour les établir, considérons le cas où les électrodes sont portées respectivement au potentiel zéro pour $\left(E_{1}\right)$ et au potentiel $V$ pour les deux autres (Fig. $3 a$ ). Dans ces conditions il ne subsiste que les éléments $R_{12}$ et $R_{31}$ du schéma équivalent en triangle de la figure $2 b$. Au sein du matériau résistant, ces deux résistances se raccordent selon une ligne de courant caractérisée par la valeur $U_{\mathbf{P}}$ de la fonction flux. Par application d'une transformation conforme $(T)$, le dispositif initial se met, dans le plan de référence, sous la forme équivalente de la figure $3 b$, où les lignes de courant sont des droites verticales et les équipotentielles des horizontales. La transformation $(T)$ est, ici, égale au produit de deux transformations de Schwarz-Christoffel, la première étant celle qui associe les cas $c$ et $d$ de la figure 1 . La seconde est telle que le segment FG de la frontière se replie verticalement sur lui-même [3]. La formulation explicite de la transformation $(T)$ nécessite la connaissance des coordonnées du point de rebroussement $P$ qui est situé entre $\mathrm{F}$ et $\mathrm{G}$. Bien que ceci soit possible, en particulier à l'aide de calculs numériques, il suffit, pour l'étude envisagée, que $(T)$ existe. La même transfor- 
mation $(T)$ appliquée au dispositif complémentaire conduit à la figure $3 c$. Dans ce cas les électrodes latérales ont un potentiel imposé tandis que $\left(E_{1}^{\prime}\right)$ est flottante.

La comparaison des figures $3 b$ et $3 c$ permet d'établir les relations suivantes, où les notations sont les mêmes que pour (3) :

$$
R_{i 0} \cdot R_{j k}^{\prime}=R_{i 0}^{\prime} \cdot R_{j k}=R_{\mathrm{E}} \cdot R_{\mathrm{P}}^{\prime}=R_{\mathrm{E}}^{\prime} \cdot R_{\mathrm{P}}=1 .
$$

Ces expressions apparaissent comme la généralisation, pour un système à trois électrodes, de la relation (2).

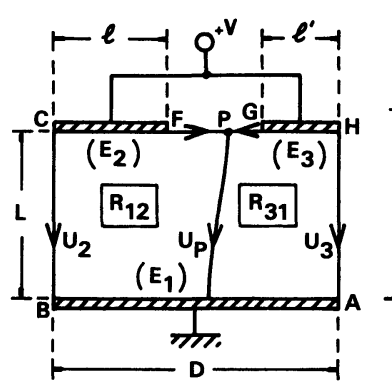

(a)

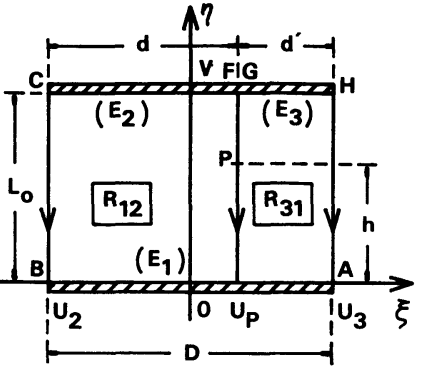

(b)

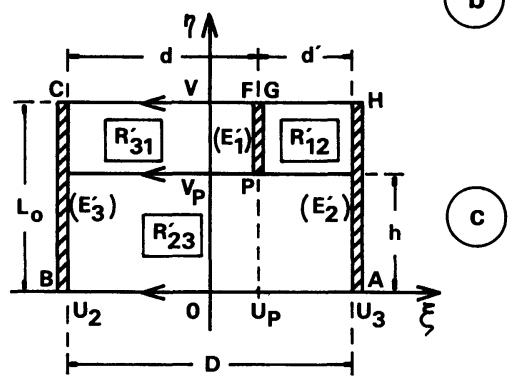

Fig. 3. - Détermination des résistances interélectrodes. a) Les électrodes $\left(E_{2}\right)$ et $\left(E_{3}\right)$ sont reliées à la même source de potentiel. Les résistances $R_{12}$ et $R_{13}$ se raccordent selon la ligne de courant $U_{\mathrm{P}}$ qui est tracée de manière arbitraire. $b$ ) Système associé au cas précédent par une transformation conforme $(T) . c)$ Dispositif complémentaire du cas précédent.

[Determination of the interelectrode resistances. $a$ ) The electrodes $\left(\mathrm{E}_{2}\right)$ and $\left(\mathrm{E}_{3}\right)$ are connected to the same voltage source. The resistors $R_{12}$ and $R_{13}$ are juxtaposed along the current line $U_{\mathrm{P}}$, the drawing of which being merely qualitative. $b$ ) Rectangle mapped onto $(a)$ by a transformation $(T)$. The current lines are vertical straight lines and the equipotentials horizontal ones. c) Complementary system of $(b)$. The electrode $\left(E_{1}^{\prime}\right)$ is not connected to a voltage source.]

En définitive, les relations (3) et (4) permettent d'obtenir les résistances des quatre schémas de la figure 2 lorsque leurs valeurs sont connues pour l'un d'entre eux. Comme la figure $3 c$ permet d'évaluer les résistances du schéma équivalent en triangle du dispositif complémentaire, il suffit de déterminer les différentes longueurs qui apparaissent sur cette figure. Cette opération comporte deux étapes, d'abord l'évaluation des dimensions latérales $D, L_{0}, d$ et $d^{\prime}$, puis l'évaluation de l'ordonnée $h$ du point de rebroussement $P$. Le caractère pratique de la méthode est souligné par l'emploi de constructions graphiques mettant en œuvre deux abaques.
4. Détermination de $R_{12}$ et $R_{31}$. - Un premier abaque permet d'obtenir les dimensions latérales de la figure $3 c$. L'exposé d'indications relatives à son élaboration justifie la détermination envisagée. La figure $4 a$ représente la résistance interélectrodes $R_{12}$, obtenue par un fractionnement du système initial de la figure $3 a$ selon la ligne de courant $U_{\mathrm{p}}$. En ayant recours à une transformation $(T)$ cette résistance se met, dans le plan de référence, sous la forme $4 b$. Les dimensions $L_{0}$ et $d$ sont, provisoirement, indéterminées. Par une transformation conforme analogue à celle qui relie les cas . $z$ et $d$ de la figure 1, la résistance est mise sous la forme équivalente $4 c$. (La transformation est étudiée dans la

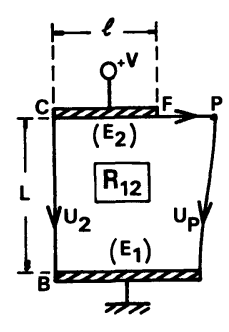

(a)

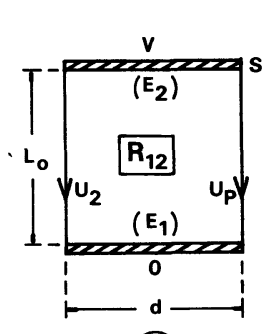

(b)

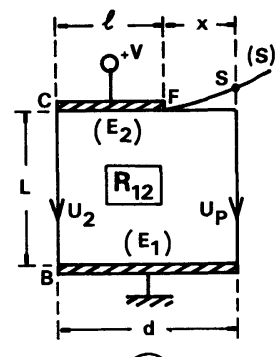

(c)
Fig. 4. - Détermination des courbes (S). $a$ ) Dispositif de résistance $R_{12}$ obtenu en partageant le rectangle de la figure $3 a$ selon la ligne de courant $U_{\mathrm{p}} . b$ ) Rectangle équivalent au cas précédent dans le plan de référence. $c$ ) Dispositif de même résistance que les cas $(a)$ et $(b)$. Les longueurs $L$ et $l$ sont les mêmes qu'en $a$ et la largeur est la même qu'en $(b)$.

[Determination of curves (S). a) System, with resistance $R_{12}$, obtained by cutting the rectangle of figure $3 a$ along the current line $\left.U_{\mathrm{p}} . b\right)$ Rectangle equivalent to $(a)$ in the reference plane. $c$ ) Rectangle with the same resistance as in $(a)$ and $(b)$. The lengths $L$ and $l$ are the same as in $(a)$ and the width is the same as in $(b)$. The point $\mathbf{S}$ is the image of the vertex $S$ in $(b)$.]

première partie de cet article.) Sur cette figure les longueurs $L$ et $l$ sont les mêmes qu'en $a$, alors que la largeur, $d$, est la même qu'en $b$. Grâce aux procédés décrits dans la première partie de l'article [1], la largeur $d$, des rectangles $b$ et $c$ de la figure 4 , et la longueur $L_{0}$, du cas $b$, peuvent se déduire de la valeur de la résistance $R_{12}$. Lorsque $R_{12}$ varie, $l$ et $L$ restant constants, l'image du sommet $S$ du rectangle de la figure $4 b$ décrit, dans le plan de la figure $4 c$, une courbe (S) d'origine $F$. Cette courbe est déterminée par la donnée du paramètre $l / L$. L'abaque de la figure 5 représente, avec des coordonnées réduites par rapport à la longueur $L$, des courbes $(\mathbf{S})$ tracées à partir d'une origine commune et correspondant à une gamme étendue de paramètres $l / L$.

Le principe de la détermination des longueurs $L_{0}$, $d$ et $d^{\prime}$ à l'aide de l'abaque de la figure 5 est le suivant. Soit un dispositif à trois électrodes quelconques (Fig. 6a). Les longueurs $D, L, l$ et $l^{\prime}$ ont des valeurs connues. Le rapport $l / L$ caractérise, sur l'abaque, la courbe (S) qui est reportée sur la figure $6 a$, à partir de l'extrémité $\mathrm{F}$ de $\left(\mathrm{E}_{2}\right)$ prise comme origine. De même, 


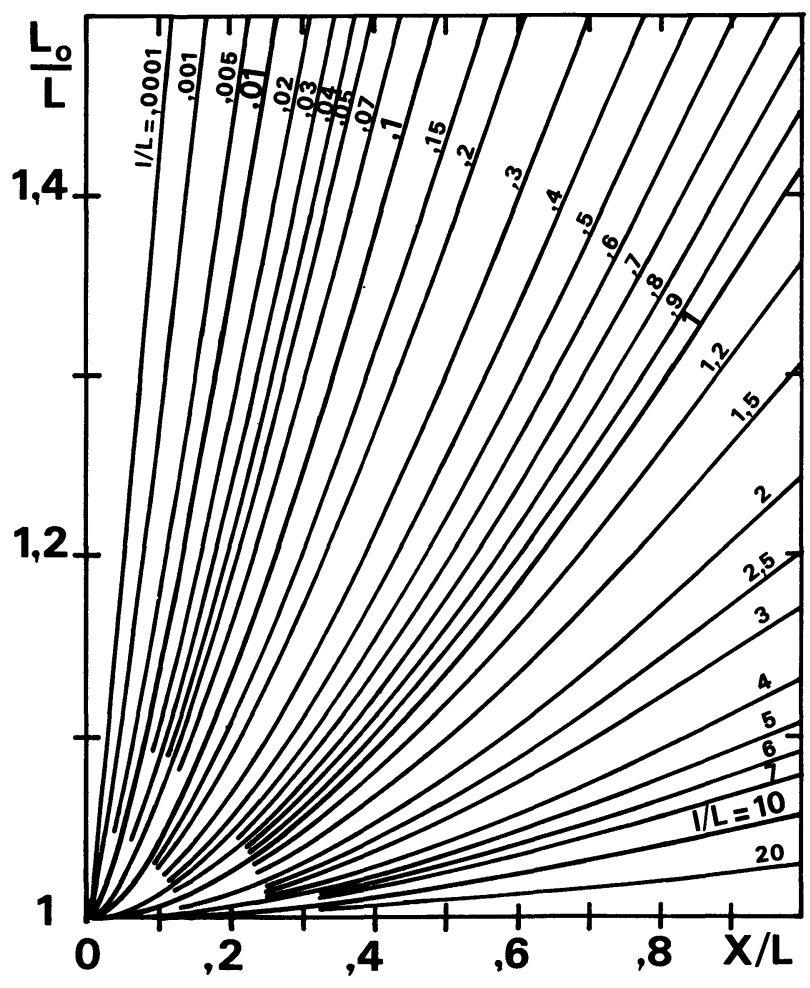

a)

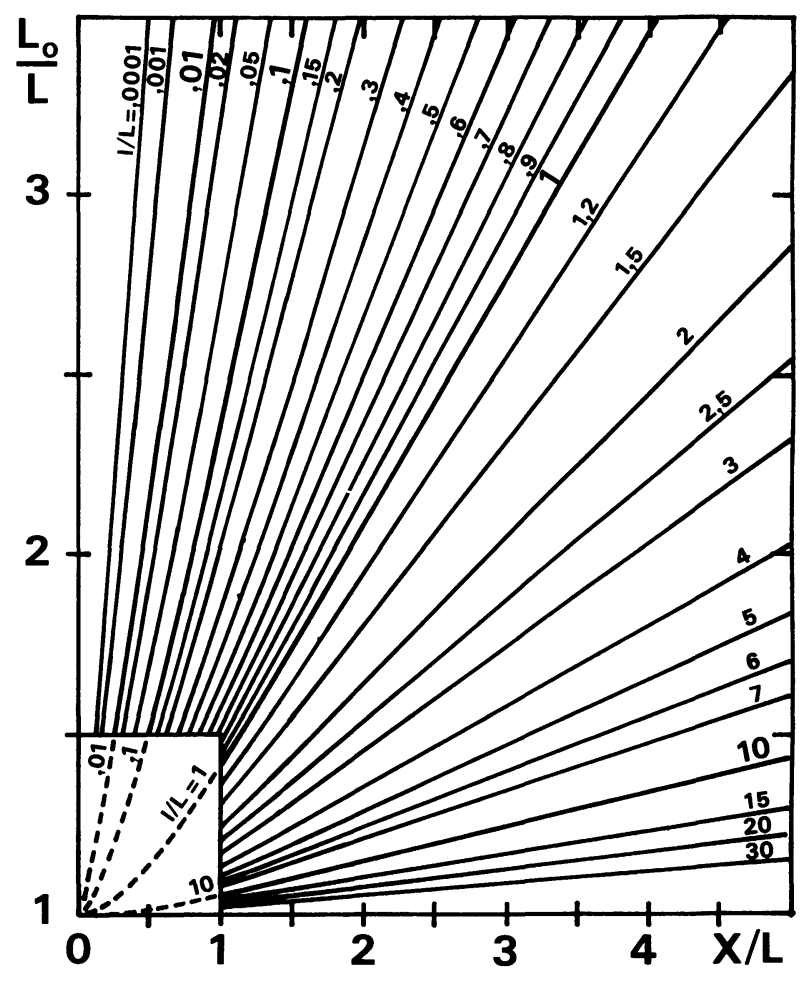

b)

Fig. 5. - Abaque de courbes (S). Les notations sont celles de la figure 4. Toutes les grandeurs sont réduites par rapport à la longueur $L$. Les courbes sont caractérisées par le paramètre $l / L$. La figure $(a)$ est un agrandissement de la zone voisine de l'origine sur la figure $(b)$.

[Chart of curves (S). The notations are those of figure 4. All the quantities are reduced with respect to the length $L$. The curves are characterized by the parameter $l / L$. The drawing $(a)$ focusses on the vicinity of the origin in $(b)$.]

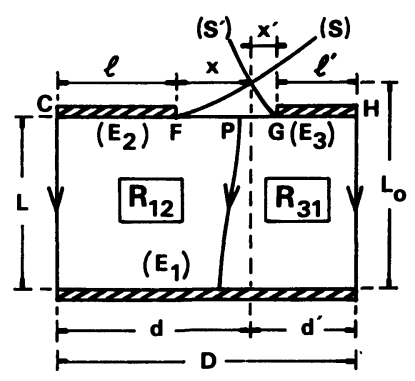

(a)

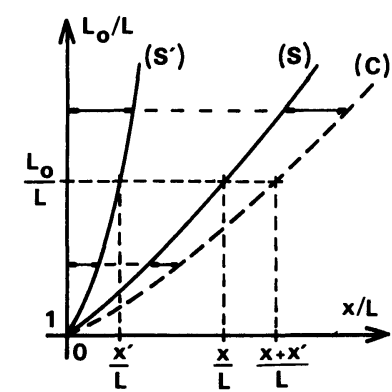

(b)
Fig. 6. - Emploi de l'abaque de la figure 5. a) Dispositif rectangulaire à trois électrodes. Les longueurs $D, L, l$ et $l^{\prime}$ sont connues. Les courbes (S) et ( $\left.\mathbf{S}^{\prime}\right)$ correspondent, sur l'abaque, aux paramètres respectifs $l / L$ et $l^{\prime} / L$. Le point d'intersection de (S) et de ( $\left.\mathbf{S}^{\prime}\right)$ détermine les longueurs $L_{0}, d$ et $d^{\prime}$ de la figure $3 b . b$ ) Détermination de $L_{0}$. La courbe (C) se déduit de (S) et ( $\left.S^{\prime}\right)$ par construction graphique. Le rapport $L_{0} / L$ cherché est l'ordonnée du point de (C) ayant pour abscisse la quantité connue $\left(x+x^{\prime}\right) / L$.

[Use of the chart of figure 5.a) Three-electrode rectangular system. The lengths $D, L, l$ and $l^{\prime}$ have known values. The curve (S) has its origin in $F$, while $G$ is the origin of $\left(S^{\prime}\right)$. The intersecting point of (S) and $\left(\mathrm{S}^{\prime}\right)$ determinates the lengths $L_{0}, d$ and $d^{\prime}$ in figure $3 b$. b) Determinations on the chart. The curves $\left(\mathbf{S}^{\prime}\right)$ and $\left(\mathbf{S}^{\prime}\right)$ correspond to the parameters $l / L$ and $l^{\prime} / L$. The curve (C) is derived from (S) and $\left(S^{\prime}\right)$ by graphical construction. The ratio $L_{0} / L$ is the ordinate for the point of $(C)$ with the known abscissa $\left(x+x^{\prime}\right) / L$. For such an ordinate, the curves (S) and $\left(\mathbf{S}^{\prime}\right)$ determinate the abscissas $x$ and $x^{\prime}$, from which the lengths $d$ and $d^{\prime}$ are deduced.] la courbe $\left(\mathbf{S}^{\prime}\right)$, de paramètre $l^{\prime} / L$, est reportée, après un retournement, à partir de l'extrémité $G$ de $\left(E_{3}\right)$. Le point d'intersection de $(S)$ et $\left(S^{\prime}\right)$ détermine les longueurs $L_{0}, d$ et $d^{\prime}$. En effet il y a, en ce point, conservation de la valeur numérique de la résistance $R_{12}$ par l'intermédiaire de la courbe (S) et conservation de $R_{31}$ par $\left(\mathbf{S}^{\prime}\right)$, ce qui détermine le dispositif de la figure $3 b$. En pratique les déterminations de $L_{0}, d$ et $d^{\prime}$ s'effectuent directement sur l'abaque. Pour cela on sélectionne les courbes $(S)$ et $\left(S^{\prime}\right)$ de paramètres respectifs $l / L$ et $l^{\prime} / L$ (Fig. $6 b$ ). Puis on trace la courbe (C) en additionnant les abscisses des points de (S) et $\left(\mathbf{S}^{\prime}\right)$ de même ordonnée $L_{0} / L$. Il faut noter que $(C)$ ne coïncide pas avec une courbe de paramètre $\left(l+l^{\prime}\right) / L$ car les résistances interélectrodes dépendent à la fois de la dimension et de la localisation des électrodes. Le point de (C) dont l'abscisse correspond à l'intervalle FG entre $\left(E_{2}\right)$ et $\left(E_{3}\right)$ permet de tracer une droite horizontale qui coupe l'axe vertical au point d'ordonnée $L_{0} / L$, ce qui détermine $L_{0}$. Les abscisses réduites $x / L$ et $x^{\prime} / L$ des intersections de cette droite avec (S) et $\left(\mathbf{S}^{\prime}\right)$ permettent ensuite d'évaluer les longueurs $d$ et $d^{\prime}$, respectivement égales à $l+x$ et $l^{\prime}+x^{\prime}$.

5. Détermination de $\boldsymbol{R}_{23}$. - Au stade actuel du processus les résistances $R_{12}$ et $R_{31}$ des figures $2 b$ et $3 b$ sont connues. Il existe deux méthodes pour évaluer la 
troisième résistance, $R_{23}$, du schéma équivalent en triangle. Celle retenue passe par l'évaluation de l'ordonnée du point de rebroussement, la seconde utilisant la suite de transformations précisées sur la figure 1. L'homographie reliant les cas $b$ et $c$ de cette figure est modifiée de façon à aboutir à une permutation circulaire des électrodes. En procédant comme ci-dessus, l'électrode centrale étant portée au potentiel zéro et les deux autres étant reliées à un potentiel commun, il est alors possible d'évaluer $R_{23}$, avec une détermination redondante pour $R_{12}$ ou $R_{31}$. Cette procédure étant relativement longue, il paraît préférable d'avoir recours à la première méthode. En effet, lorsque l'ordonnée $h$ du point de rebroussement est connue, toutes les résistances sont connues et la résistance $R_{23}$ est telle que

$$
R_{23}=\left(\frac{L_{0}-h}{h}\right)\left(R_{12}+R_{31}\right) .
$$

La détermination de $h$ est fondée sur l'emploi de l'abaque de la figure 8 . Afin de justifier cette détermination il paraît nécessaire de donner quelques indications sur l'élaboration de l'abaque. On procède à la

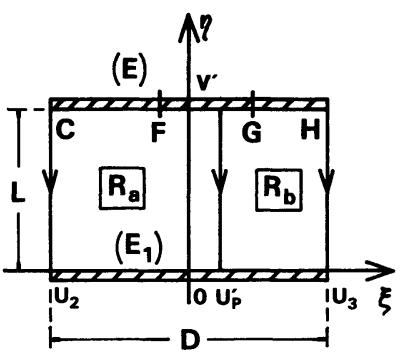

(a)

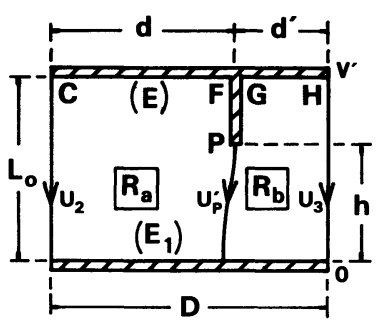

(b)

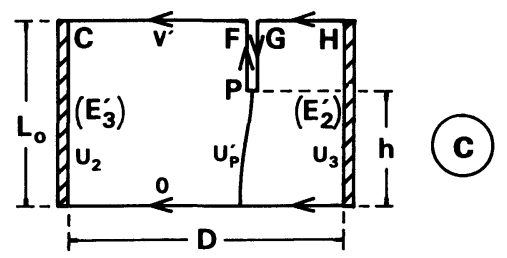

Fig. 7. - Systèmes rectangulaires à deux électrodes. a) Dispositif à deux électrodes obtenu en métallisant l'intervalle FG entre les électrodes $\left(\mathrm{E}_{2}\right)$ et $\left(\mathrm{E}_{3}\right)$ de la figure $\left.3 a . b\right)$ Rectangle issu du cas précédent par application de la transformation $(T)$ de la figure 3. L'électrode supérieure (E) présente une indentation selon le segment FPG. La ligne de courant $U_{\mathrm{P}}^{\prime}$ est représentée de manière arbitraire car son tracé réel n'est pas connu. c) Dispositif complémentaire du cas précédent. L'indentation de la figure (b) est remplacée par une entaille isolante.

[Two-electrode rectangular systems. a) Two-electrode rectangle resulting from the metallization of the interval FG between the electrodes $\left(\mathrm{E}_{2}\right)$ and $\left(\mathrm{E}_{3}\right)$ in figure $3 a$. The current line $U_{\mathrm{P}}^{\prime}$ halves the system into rectangles of respective resistances $R_{\mathrm{a}}$ and $R_{\mathrm{b}} . b$ ) System derived from $(b)$ by means of the transformation $(T)$ of figure 3 . The dimensions are the same as in figure $3 b$. The upper electrode (E) has an indentation along the twofold segment FPG. The current line $U_{\mathrm{P}}^{\prime}$ is arbitrarily drawn, its actual trace being unknown. c) Complementary system of $(b)$. The previous indentation is now an insulating notch.] métallisation, virtuelle, de l'intervalle entre les électrodes $\left(E_{2}\right)$ et $\left(E_{3}\right)$ afin de constituer une électrode unique (E). Le plan du dispositif à deux électrodes ainsi obtenu (Fig. 7a) coïncide alors avec le plan de référence $(\xi, \eta)$. On choisit arbitrairement pour les lignes de courant $U_{2}$ et $U_{3}$ les mêmes valeurs que sur la figure 3. Il en résulte que la ligne de courant issue de $P$ et la différence de potentiel interélectrodes ont de nouvelles valeurs, respectivement $U_{\mathrm{P}}^{\prime}$ et $V^{\prime}$. Le dispositif, dont la résistance totale est égale à $L / D$, est partagé en deux rectangles, de résistances $R_{\mathrm{a}}$ et $R_{\mathrm{b}}$, par la ligne de courant $U_{\mathrm{P}}^{\prime}$. La transformation $(T)$ qui associait les cas $a$ et $b$ de la figure 3 conduit ici au rectangle de la figure $7 b$ où l'électrode $(E)$ présente l'indentation FPG. Il faut noter que les longueurs $D$, $L_{0}, d, d^{\prime}$ et $h$ ont les mêmes valeurs que sur la figure $3 b$, mais que le trajet de la ligne de courant $U_{\mathbf{P}}^{\prime}$ n'est pas connu. Pour le dispositif complémentaire (Fig. 7c) l'électrode flottante $\left(\mathrm{E}_{1}^{\prime}\right)$ de la figure $3 c$ est remplacée par une entaille isolante. On considère alors le cas particulier où les longueurs $d$ et $d^{\prime}$ de la figure $7 b$ sont égales. Les différents rectangles de la figure 7 présentent ainsi une symétrie d'axe vertical et la ligne de courant $U_{\mathrm{P}}^{\prime}$ coïncide avec l'axe des ordonnées. Les résistances, égales, $R_{\mathrm{a}}$ et $R_{\mathrm{b}}$ de la figure $7 \mathrm{~b}$ correspondent donc à des dispositifs rectangulaires à deux électrodes qui peuvent être étudiés par les procédés décrits dans la première partie de l'article. Cependant, dans le cas présent, s'il est nécessaire de connaître les dimensions de $R_{\mathrm{a}}$, la valeur de cette résistance n'est pas utile. Comme la première partie est orientée vers la détermination des valeurs de résistances, il a paru préférable d'établir un nouvel abaque fournissant, par simple lecture, la relation entre les longueurs $L, L_{0}$, $D$ et $h$ (Fig. 8). Cet abaque correspond à un rapport $d / D$ égal à $1 / 2$. Il peut aussi être utilisé lorsque $œ$ rapport est égal à zéro (ou un). En effet, dans ces conditions, l'indentation FPG de la figure $7 b$ recouvre une des faces latérales du rectangle, la ligne de courant $U_{\mathrm{P}}^{\prime}$ prend la place de $U_{2}$ (ou $U_{3}$ ) et il ne subsiste que la résistance rectangulaire à deux électrodes $R_{\mathrm{b}}$ (ou $R_{\mathrm{a}}$ ). Les dimensions de cette résistance sont donc fournies par l'abaque de la figure 8 en divisant par 2 le paramètre $L_{0} / D$. En comparant les courbes tracées sur l'abaque, il apparaît que le rapport $L / L_{0}$ varie peu lorsque, pour $h$ constant, $d / D$ passe de la valeur 0 à la valeur $1 / 2$, c'est-à-dire lorsque le paramètre $L_{0} / D$ varie du simple au double. On peut noter qu'une caractérisation plus précise des variations de $L / L_{0}$ est fournie par les courbes tracées dans l'encadré de la figure 8 , mais cette étude est différée jusqu'à la fin du présent paragraphe.

La remarque précédente, concernant les faibles variations de $L / L_{0}$, conduit à une évaluation approximative de la relation entre les longueurs $h$ et $L$, de la figure 7 , lorsque le rapport $d / D$ est quelconque. Il y a lieu de distinguer les deux types de problèmes pour lesquels il faut établir cette relation entre $h$ et $L$. Dans le premier cas, comme par exemple lors de l'évaluation 


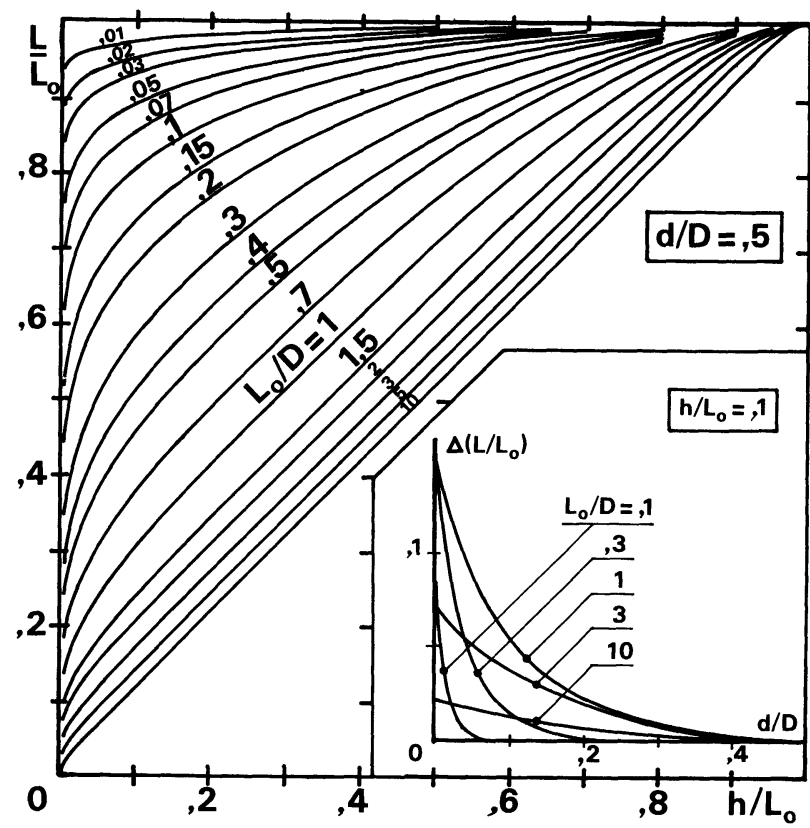

Fig. 8. - Abaque reliant $L$ et $h$. Les notations sont celles de la figure 7. Les longueurs $L$ et $h$ sont réduites par rapport à $L_{0}$. Les courbes sont caractérisées par le paramètre $L_{0} / D$. Les courbes dans l'encadré représentent l'accroissement du rapport $L / L_{0}$ lorsque $d / D$ varie de $1 / 2$ à 0 . La longueur réduite $h / L_{0}$ correspond à la zone de variations maximales de $L$ en fonction de $d$.

[Chart relating $L$ to $h$. The notations are the same as in figure 7. The lengths $L$ and $h$ are reduced with respect to $L_{0}$. The curves are characterized by the parameter $L_{0} / D$. The chart applies to symmetrical rectangles $(d / D$ equal to $1 / 2)$. The curves within the inset show the increase of $L / L_{0}$ as $d / D$ varies from $1 / 2$ to 0 . These curves are drawn for a reduced length $h / L_{0}$ chosen within the area where the variations of $L$ against $d$ are the largest.]

de $R_{23}$, la longueur $L$ est connue et il faut déterminer $h$. Au contraire, dans le second cas, qui correspond à des applications évoquées au paragraphe suivant, il faut déterminer $L$ à partir de la valeur de $h$. Considérons tout d'abord cette seconde éventualité. La valeur de $L / L_{0}$ dépend essentiellement de l'ordonnée $h$ du point $P$, alors qu'elle est peu affectée par les variations de l'abscisse de $\mathrm{P}$, repérée par $d / D$. Par conséquent le remplacement de la ligne de courant réelle, et inconnue, $U_{\mathrm{P}}^{\prime}$ de la figure $7 b$ par une ligne de courant fictive verticale (Fig. 9a) n'introduit qu'une faible erreur dans la détermination de la longueur $L$ associée à $h$ (cette erreur est évaluée en annexe). Les résistances réelles $R_{\mathrm{a}}$ et $R_{\mathrm{b}}$ sont ainsi remplacées par des résistances rectangulaires $R_{\alpha}$ et $R_{\beta}$. La résistance $R_{\alpha}$ est prise isolément de façon à constituer une géométrie caractérisée par un rapport $d / D$ égal à un, ce qui permet l'emploi de l'abaque de la figure 8 . Il en résulte une répartition d'électrodes en coïncidence avec deux côtés opposés d'un rectangle. Les mêmes transformations sont effectuées sur $R_{\beta}$, puis $R_{\alpha}$ et $R_{\beta}$ sont associées (Fig. $9 b$ ) afin de reconstituer un dispositif semblable à celui de la figure $7 b$. Par suite de la modification apportée au tracé de la ligne de courant $U_{\mathrm{P}}^{\prime}$, la longueur $L$ de la figure $9 b$ n'est pas rigoureusement égale à la longueur $L$

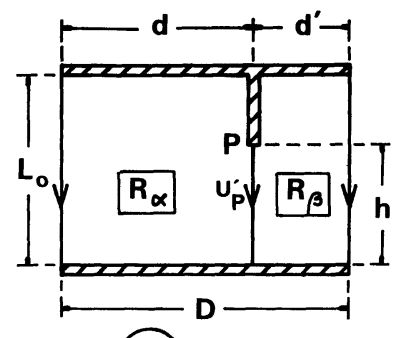

(a)

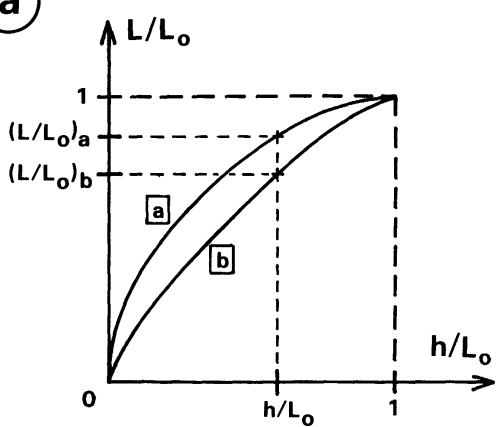

Fig. 9. - Utilisation de l'abaque de la figure 8. a) Dispositif issu de la figure $7 b$ en adoptant un tracé rectiligne fictif pour la ligne de courant $U_{\mathrm{P}}^{\prime} . b$ ) Système équivalent au cas précédent. $c$ ) Détermination de $L$ à l'aide de l'abaque de la figure 8 .

[Use of the chart of figure 8. $a$ ) Rectangle derived from figure $7 b$ when the current line $U_{\mathbf{P}}^{\prime}$ is arbitrarily drawn as a straight line. $b$ ) System equivalent to $(a) . c)$ Determining $L$ by means of the chart of figure 8 . The two rectangles in $(a)$ are considered as independent systems. For the left one, the $d / D$ value is unity and the parameter of the associated curve, on the chart, is $L_{0} / 2 d$. The quantity $\left(L / L_{0}\right)_{\mathrm{a}}$ is related, through the curve $(a)$, to the ratio $h / L_{0}$. In the same way the second geometry leads to $\left(L / L_{0}\right)_{\mathrm{b}}$. Thereafter the length $L$ is given by equation (6).] de la figure $7 a$, mais comme il a été indiqué ci-dessus, l'écart entre ces deux longueurs est relativement faible.

La détermination, approximative, de $L$, à l'aide de l'abaque de la figure 8 , est représentée sur la figure $9 c$. Les deux rectangles de la figure $9 a$ sont traités comme des systèmes indépendants. La courbe (a), sur l'abaque, est relative au rectangle de résistance $R_{\alpha}$. Pour ce rectangle, pris isolément, le rapport $d / D$ vaut un et le paramètre de la courbe (a) est égal à $L_{0} / 2 d$. Pour l'abscisse, connue, $h / L_{0}$ la courbe (a) détermine une valeur particulière de l'ordonnée $L / L_{0}$ qui est notée $\left(L / L_{0}\right)_{a}$. De manière identique dans le cas de $R_{\beta}$, la courbe (b), de paramètre $L_{0} / 2 d^{\prime}$, détermine la valeur $\left(L / L_{0}\right)_{b}$. La longueur $L$ cherchée est obtenue en associant les rectangles $R_{\alpha}$ et $R_{\beta}$ de façon à aboutir à la figure $9 b$. On obtient ainsi :

$$
\frac{L_{0}}{L}=\frac{d}{D}\left(\frac{L_{0}}{L}\right)_{\mathrm{a}}+\frac{d^{\prime}}{D}\left(\frac{L_{0}}{L}\right)_{\mathrm{b}}
$$

Dans le cas opposé au précédent, c'est-à-dire celui pour lequel on détermine $h$ à partir de $L$, le procédé de résolution fait appel à la relation (6). Pour cela on sélectionne sur l'abaque de la figure 8 les courbes de paramètres, connus, $L_{0} /(2 d)$ et $L_{0} /\left(2 d^{\prime}\right)$. Pour une 
valeur arbitraire de $h$, ces courbes fournissent les rapports $\left(L_{0} / L\right)_{\mathrm{a}}$ et $\left(L_{0} / L\right)_{\mathrm{b}}$ (Fig. 9c). La relation (6) conduit alors à une valeur du rapport $L_{0} / L$. Comme ce rapport est connu a priori, le processus est repris en modifiant la valeur de $h$ jusqu'à ce que les valeurs calculée et réelle de $L_{0} / L$ coïncident, ce qui fournit la valeur de $h$ cherchée. (Cette détermination peut s'effectuer graphiquement en construisant la courbe de $L_{0} / L$ en fonction de $h$.)

Le procédé approximatif de détermination de $L$ a été utilisé pour caractériser la variation de cette grandeur lorsque le point de rebroussement $\mathrm{P}$ (Fig. $7 b$ ) se déplace horizontalement, c'est-à-dire lorsque $d$ varie avec $h$ constant. Ces variations, réduites par rapport à $L_{0}$ et notées $\Delta\left(L / L_{0}\right)$, sont représentées dans l'encadré sur l'abaque de la figure 8 . La valeur choisie pour $h$ correspond au domaine où $L / L_{0}$ varie dans les plus grandes proportions. A l'examen des courbes il apparaît que la variation globale de $L$ est faible et que l'essentiel de cette variation s'effectue dans le domaine voisin de l'origine, c'est-à-dire lorsque l'entaille FPG de la figure $7 c$ est proche d'une face latérale du rectangle. Cette constatation renforce la validité du procédé approximatif de détermination de $L$ (ou de $h$ ), puisque le remplacement de la figure $7 b$ par la figure $9 a$ ne peut avoir qu'une faible incidence sur la valeur de cette grandeur.

6. Applications. - Une première application découle de la relative indépendance de $L$ vis-à-vis de $d$. Pour cela on considère que la figure $7 c$ représente un élément de circuit intégré. L'entaille FPG est pratiquée dans la couche conductrice, par exemple à l'aide d'un faisceau laser, afin d'augmenter la valeur numérique de la résistance. L'étude montre que, à condition d'éviter les zones proches des électrodes, la localisation de l'entaille n'a qu'une importance secondaire et que la valeur obtenue pour la résistance dépend essentiellement de l'extension de cette entaille. En outre, les courbes de la figure 8 montrent que l'augmentation de résistance peut difficilement dépasser un facteur 10 , ce qui limite l'application du procédé à des ajustages précis de résistances.

Précédemment, les résistances des schémas équivalents de la figure 2 ont été obtenues à partir d'une géométrie donnée. Une application inverse est la détermination de la répartition d'électrodes correspondant à un schéma équivalent donné. En utilisant, si nécessaire, les relations (3) et (4) ce schéma est mis sous la forme de la figure $2 b$. La longueur $h$ se déduit de la relation (5)

$$
h=L_{0} \cdot\left(\frac{R_{12}+R_{31}}{R_{\mathrm{P}}}\right) .
$$

Toutes les dimensions de la figure $3 b$ sont alors connues (après avoir choisi arbitrairement l'une d'entre elles). Initialement, le dispositif est recherché sous la forme des figures $3 a$ ou $6 a$. Il faut donc déterminer la largeur $L$ du barreau et les longueurs respectives $l$ et $l^{\prime}$ des électrodes $\left(E_{2}\right)$ et $\left(E_{3}\right)$. En ce qui concerne $L$, il a été vu plus haut que l'emploi combiné des constructions de la figure $9 c$ et de la relation (6) détermine cette grandeur. Enfin les longueurs $l$ et $l^{\prime}$ sont obtenues graphiquement. Pour cela une droite horizontale, d'ordonnée $L_{0} / L$, est tracée sur l'abaque de la figure 5. Les points d'intersection avec les courbes de l'abaque sont reportés sur un graphique auxiliaire où l'abscisse et l'ordonnée sont respectivement égales à $l$ et à $x+l$, c'est-à-dire $d$ (cf. Fig. 6a). La courbe ainsi tracée fournit les longueurs $l$ et $l^{\prime}$ associées aux quantités connues $d$ et $d^{\prime}$. Le dispositif est alors connu sous une forme semblable à celle de la figure $1 d$. En ayant ensuite recours aux transformations de la figure 1 , le dispositif peut être mis sous une forme rectangulaire quelconque. Il apparaît ainsi qu'outre les valeurs des trois résistances il est possible de fixer a priori diverses données géométriques comme, par exemple, les dimensions du rectangle.

Une application, aisément prévisible, concerne la détermination de la résistance de dispositifs à deux électrodes présentant une indentation ou une entaille (cf. Fig. 7). Quelle que soit la disposition réelle des électrodes (Fig. 10a), la géométrie donnée est mise sous la forme équivalente $10 \mathrm{~b}$. En procédant comme cidessus, pour la figure 9 , le système prend la forme $10 c$.

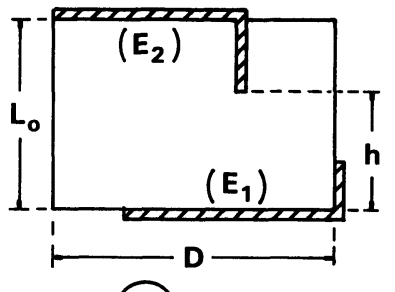

a)
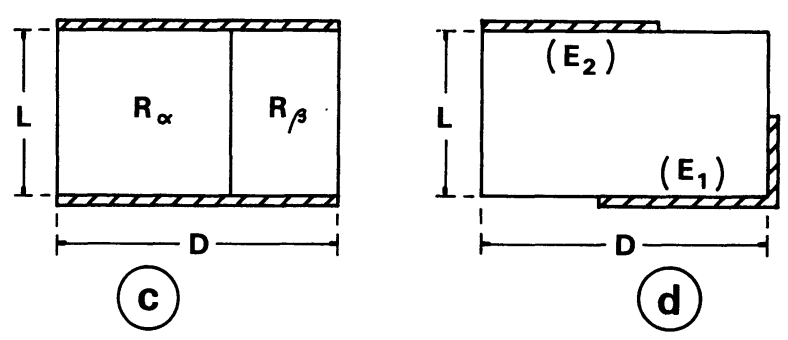

Fig. 10. - Systèmes à indentation. a) Dispositif rectangulaire à deux électrodes quelconques. L'électrode $\left(\mathrm{E}_{2}\right)$ présente une indentation. $b$ ) Rectangle associé au cas précédent. Tout en conservant l'indentation, les électrodes sont modifiées afin d'obtenir un rectangle semblable à celui de la figure $9 a$. c) Rectangle déduit du cas $b$ par le procédé de la figure 9. $d$ ) Dispositif rectangulaire équivalent au cas $a$. Les dimensions sont les mêmes qu'en $c$ mais les électrodes correspondent à celles du premier cas. Les dispositifs $a$ et $d$ présentent la même résistance interélectrodes.

[Systems with indentations. a) Two-electrode system with an indentation on the electrode $\left(\mathrm{E}_{2}\right) . b$ ) Rectangle derived from $(a)$. While the indentation is held in place, the electrodes are altered in order to obtain a rectangle similar to figure $9 a . c$ ) Rectangle derived from (b) by the process of figure $9 . d$ ) System equivalent to $(a)$. The dimensions are the same as in $(c)$ but the electrodes correspond to $(a)$. The interelectrode resistance has the same value in $(a)$ and $(d)$.] 
Enfin le rétablissement des électrodes initiales conduit à un dispositif à deux électrodes (Fig. 10d) qui peut être étudié par les procédés décrits dans la première partie de l'article. Il faut noter que les coordonnées de points quelconques de la frontière, par exemple les extrémités de $\left(E_{1}\right)$ pour le cas considéré, peuvent être déterminées à l'aide des transformations de la figure 1 . En complétant la figure $10 a$ par symétrie, puis répétition périodique, on obtient différents dispositifs ayant une structure en peignes imbriqués dont la résistance s'évalue par la méthode précédente. De même, en complétant par symétrie la figure $7 c$, il est possible de déterminer la résistance de dispositifs possédant deux entailles symétriques ou bien une entaille interne comme, par exemple, un bac parallélépipédique dans lequel est plongée une lame isolante.

L'étude de systèmes à quatre électrodes latérales quelconques peut être envisagée par une généralisation des procédés décrits dans cet article [4], mais sa mise en œuvre nécessite un assez grand nombre de calculs préparatoires. Par contre, ainsi qu'il apparaît en complétant par symétrie les figures $2 a$ et $2 d$, l'étude de dispositifs à quatre électrodes présentant un plan de symétrie constitue une application directe des travaux précédents.

7. Conclusion. - Les travaux, qui viennent d'être décrits, établissent la relation entre résistances interélectrodes et caractéristiques géométriques pour des dispositifs rectangulaires à trois électrodes quelconques, à deux électrodes avec indentation ou bien encore à quatre électrodes présentant un plan de symétrie. Les déterminations sont relativement simples et rapides puisqu'elles reposent essentiellement sur des procédés graphiques. Le corollaire de cet aspect pratique est une précision nécessairement limitée des résultats. La recherche d'une précision accrue exige de remplacer les études graphiques par des déterminations analytiques (qui ont d'ailleurs été utilisées pour tracer les abaques des figures 5 et 8 ). Ce gain de précision paraît toutefois d'un intérêt médiocre pour plusieurs raisons. Tout d'abord la mise en œuvre de procédés numériques est moins simple et moins rapide que celle des méthodes graphiques, notamment dans le cas d'un calcul non répétitif. Ensuite, ce gain ne se justifie que si les tolérances de fabrication permettent un usinage de qualité suffisante. Enfin, il faut remarquer qu'au voisinage des extrémités d'électrodes, lorsqu'elles ne coïncident pas avec un sommet du rectangle, ainsi qu'au voisinage des points de rebroussement, il y a une concentration des lignes de courant. Il en résulte un échauffement local du matériau qui modifie la valeur des résistances interélectrodes. En conséquence, pour des études à finalité pratique, les déterminations graphiques devraient généralement s'avérer suffisantes.

En envisageant le recours à des transformations conformes, et en notant que l'étude, limitée ici au cas de résistances électriques, peut être étendue aux domaines de la physique où une grandeur vérifie l'équation de Laplace, il apparaît que ces travaux ont un vaste champ d'applications.

Annexe. - La relation entre les longueurs $L$ et $h$ a été obtenue par un procédé approximatif qui semble suffisant pour des applications pratiques. Il est toutefois possible d'obtenir une relation rigoureuse. Pour cela considérons le rectangle de la figure $11 a$ où toutes les dimensions sont connues. Il lui correspond le dispositif $11 b$ où seule la longueur $h$ est indéterminée. Ces deux systèmes présentent la même résistance équivalente, $R_{\mathrm{e}}$, égale à l'association en parallèle de $R_{12}$ et $R_{31}$. Lorsqu'on fait subir au point $\mathrm{P}$, et à la ligne de courant $U_{\mathrm{P}}$, une translation dans le plan de la figure $11 b$, les résistances $R_{12}$ et $R_{31}$ sont modifiées mais $R_{\mathrm{e}}$ garde une valeur constante. Lorsque la translation est telle que la ligne de courant $U_{\mathbf{P}}$ passe par le centre du rectangle, les rectangles présentent une symétrie par rapport à cette droite. La figure initiale est alors équivalente à la figure $11 c$ où la longueur totale des électrodes $\left(\mathrm{E}_{2}\right)$ et $\left(\mathrm{E}_{3}\right)$, la largeur $D$, ainsi que la résistance $R_{\mathrm{e}}$, ont les mêmes valeurs qu'en $a$. Par contre la longueur $L^{\prime}$ est différente de $L$. Comme la résistance $R_{\mathrm{e}}$ a une valeur connue, les procédés exposés dans la première partie de l'article permettent de déterminer $L^{\prime}$. La métallisation de l'intervalle entre $\left(E_{2}^{\prime \prime}\right)$ et $\left(E_{3}^{\prime \prime}\right)$ crée alors sur la figure $11 b$ une indentation médiane
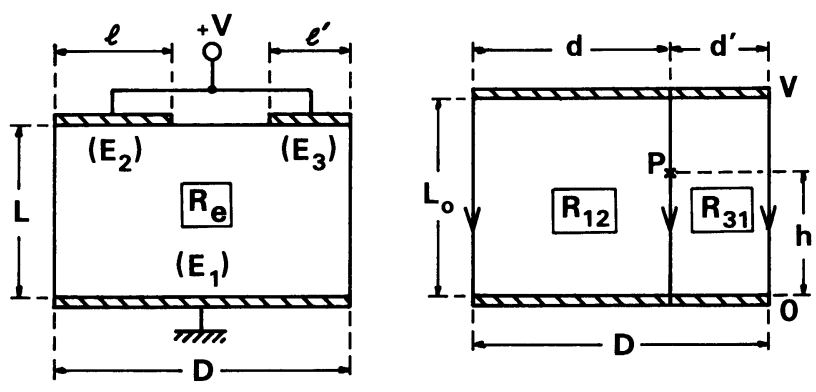

(a)
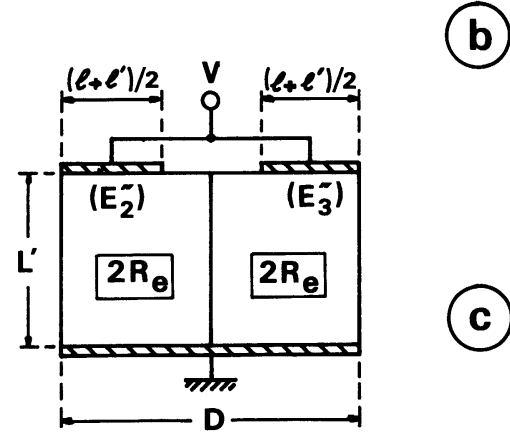

Fig. 11. - Relation entre $h$ et $L . a$ ) et $b$ ) Dispositifs semblables aux cas $a$ et $b$ de la figure 3. Ils présentent la même résistance équivalente $R_{\mathrm{e}} \cdot c$ ) Dispositif symétrique à trois électrodes. La longueur totale des électrodes a la même valeur qu'en $(a)$.

[Relation between $h$ and $L . a$ ) and $b$ ) Rectangles similar to the cases $(a)$ and $(b)$ in figure 3. Both systems have the same equivalent resistance $R_{\mathrm{e}}$. c) Three-electrode symmetrical system. The overall length of the electrodes is the same as in $(a)$. The length $L^{\prime}$ is such that the equivalent resistance is the same as in $(a)$ or $(b)$. The relation between $h$ and $L^{\prime}$, obtained from the chart of figure 8 , leads to the relation between $h$ and $L$.] 
qui pénètre jusqu'à un point de même ordonnée $h$ que le point $P$ initial car la résistance $R_{23}^{\prime}$ de la géométrie complémentaire (cf. Fig. 3c) n'est pas modifiée par la translation de P. La valeur de $h$ se déduit alors de celle de $L^{\prime}$, par exemple à l'aide de l'abaque de la figure 8 . L'écart relatif maximal entre les déterminations de $h$ par la méthode précédente et par la méthode approximative est de $5 \%$.

\section{Bibliographie}

[1] Morand, A., Robert, J., Revue Phys. Appl. 15 (1981).

[2] Durand, E., Electrostatique 2 (Masson, Paris) 1966, p. 273.

[3] Morand, A., Thèse, Paris XI-Orsay (1980) 177-181.

[4] Id. [3], p. 201-205. 